\title{
Graphical Interfaces for Dynamic Supervision for Failures Prognosis using the AI-PLC Combinatorial Approach: The Case Study of Cameroon Breweries Mill
}

\author{
Timothee KOMBE*and Sandra NZENEU \\ Laboratory of Energy, Materials, Modeling and Methods \\ Doctoral Training Unit of the Sciences of the Engineer \\ Department of Automotive Technology \\ University of Douala \\ PO Box 8698 Douala, Cameroon
}

\begin{abstract}
The increasing complexity of industrial systems and the increasingly severe operating constraints have forced specialists to design, specify and operate modern industrial processes. These evolutions thus imply the development of "intelligent" supervisory and prognostic systems, for the improvement of the control of the processes and the realization of the maintenance actions. This article presents our contribution in the study and the development of an interface of supervision and failures prediction of the Carbomill (malt mill) of the Breweries of Cameroon. The methodological approach is based on the design of a graphical interface made under Vijeo and controlled by an PLC, programmed on Unity Pro XL and the use of an ANFIS neuro-fuzzy network as a prediction tool. The expected results lead at the end of the learning on the evaluation by an RMSE cost function of 0.2142.
\end{abstract}

Key words: Graphical interface, Dynamic Supervision, Prognosis, Programmable Logic Controller, Artificial Intelligence, Neuro-fuzzy networks.

\section{INTRODUCTION}

The need for human-machine communication has been felt in the industries, with the aim of optimizing performance, ensuring the safety of equipment and the safety of people. To this end, the technological evolution in recent years has allowed industrial processes to grow in size and complexity, with the rise of automation. In these circumstances, the place of man in the system, its role, its degree of autonomy, its professional qualifications required, are changed in a major way. Indeed, this evolution results in a quantity of information more and more important, that the human operator must treat, in order to carry out its tasks.

Thus, the implementation of so-called "intelligent" technologies will provide the operator with useful information, clear and concise, to assist in the decision-making maintenance actions to be performed. To this end, faced with the regular failures encountered by the Carbomill (malt mill) of the mill of the Breweries of Yaounde (Cameroon), a proposed solution is to set up a management system of the process of manufacturing beer. Its main activities are: the dynamic supervision of significant parameters, the prediction of the temperature evolution of the mill crushing chamber and the classification of the predicted temperature in operating modes.

\section{INDUSTRIAL GRAPHICAL INTERFACES}

Intermittent mill failures and the difficult operating conditions encountered at the flour mill (domain ensuring the processing of raw materials for the manufacture of beer) of the Breweries of Yaounde (Cameroon) impact the availability of production equipment, thus influencing the process of processing the raw materials used for making beer. The various problems thus show the limit of application of the usual methods of conventional surpervision which do not take into account the notion of "anticipation of failures". Hence the interest of integrating Artificial Intelligence (AI) [1], [2], into the supervision tools, in order to optimize the management of industrial processes and to enable the prognosis of failures [3] and [4]. The combination with the Programmable Logic Controller (PLC) is an undeniable additional contribution. It makes it possible to benefit from the great capacity of communication and data processing which it has. 
The most recent tools are those dedicated to online monitoring and improve equipment maintenance by anticipating failures, thus making it possible to optimize the planning of maintenance operations in order to avoid costly of immobilization periods [5]. An online prediction makes it possible to control an industrial installation at any time so that preventive and / or corrective measures can be taken [6]. Predictive maintenance of complex dynamic systems is part of a relatively new field of research [5]. It is based on supervision tools and failure prognosis sensitive to process evolutions [7]. In fact, the operators in charge of the management of industrial processes must be informed of the degradation of the behavior of the systems in relation to the expected nominal behavior, but also of the expected change of behavior over a given time horizon, so as to be able to plan and implement actions.

Thus, unlike passive graphic interfaces that provide information on the state of evolution of industrial systems and generate gross alarms after the appearance of faults, the most recent types of graphical interfaces integrate the notion of intelligence so as to assist the user operator for diagnosis and decision making. There are two types of "intelligent" interfaces:

- Advanced graphical interfaces: they are characterized by "intelligent" decision support systems integrating a user interface management system [8]. There are several types, namely: flexible interfaces, interfaces tolerant to human errors, adaptive interfaces and expert interfaces. All these interfaces characterize a system of assistance in the cognitive tasks of the operator providing decision support.

- Intelligent interfaces: they allow to improve the concept of advanced interface with the application of the tools of the artificial intelligence, for the resolution of the problems of detection and diagnosis encountered during the conduct of the industrial processes. There are mainly two types of intelligent interfaces that can be mentioned: assistant operators where the interface must be able to take personal initiatives, for example by testing parameters or predicting deductible failures but the human operator remains the decision maker final. Intelligent agents inspired by the field of distributed problem solving, and more particularly of work carried out in Artificial Intelligence Distributed by [9], which groups the disciplines aimed at the design of a set of entities reproducing human intellectual activities.

\section{INDUSTRIAL DYNAMIC SUPERVISION FOR THE PROGNOSIS OF FAILURES}

The goal of supervision is to control and monitor in real time the evolution of significant parameters of a system, so as to highlight their degradation [10]. Industrial supervision is made of two functional sub-units: The control, which is the set of operations allowing to act directly on the process via a Programmable Logic Controller (PLC). Indeed, from the operating parameters entered in setpoint, we observe an update of the outputs for the proper functioning of the system. Surpervising, which is an informational device providing information on the state of evolution of the system, by providing indicators.

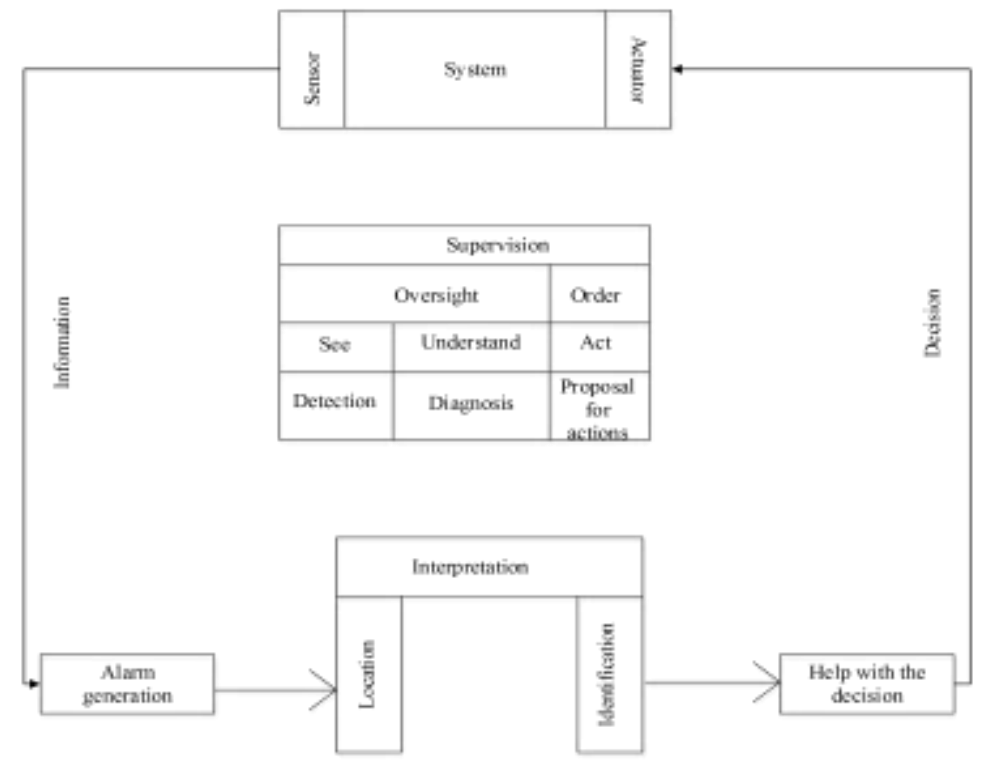

Figure 1: Functions of the supervision of a complex system 
Increasingly severe operating constraints, the increasing complexity of equipment and a very demanding continuity of service push the Breweries of Cameroon to improve the control of the operating status of their plant. Since the maintenance function does not provide optimal performance in view of the technical and economic resources involved, the optimization of the maintenance policy is proving to be an important point. For this purpose, previsionnal maintenance, which is increasingly ambitious, makes it possible to control efficiently the proper functioning of a process and to avoid costly of immobilization periods of equipment.

The mill is the first step in the process of making beer. For this, the availability of equipment and the real-time control of system parameters are very important to avoid causing heavy unavailability and thus affect the rest of the production process. The lack of real-time monitoring of the parameters of the mill, the difficulty of detecting failures and the difficulty of operating the equipment are at the origin of many failures, the most important of which are listed on the malt mill.

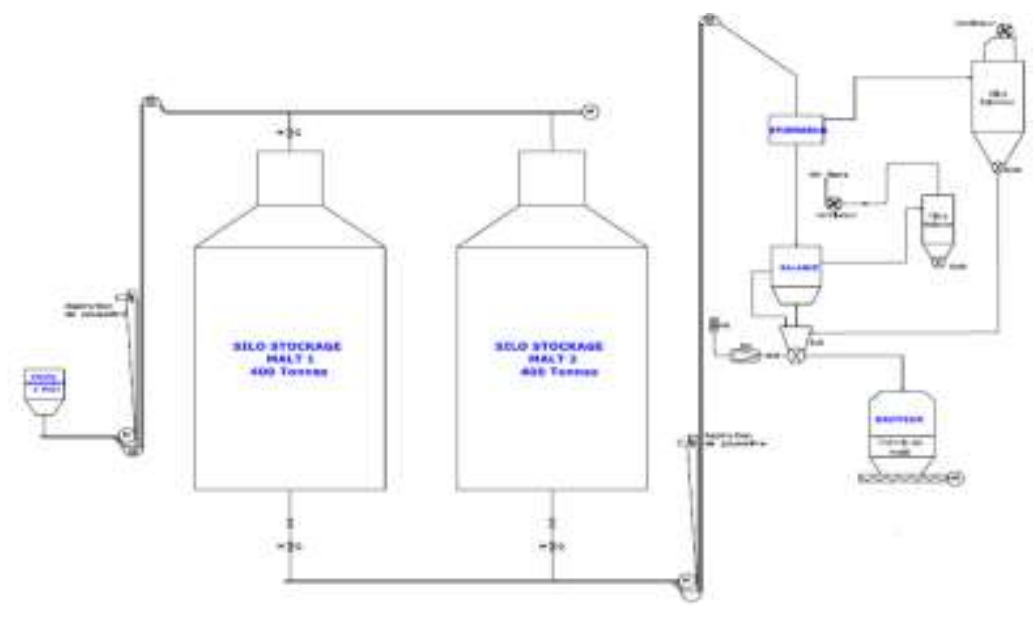

The malt mil

Figure 2: Functional diagram of the milling malt circuit atmosphere, in order to produce the desired flour for milling. Abnormal mill behavior is generally observed, caused by degradation of upstream equipment parameters or its own components. Indeed, during the process, we generally observe a malt jam upstream of the mill, causing its operation to empty. Thus the hammers hit the shock plates and are the cause of a phenomenon of friction and wear of mechanical parts, causing an increase in the temperature of the crushing chamber. This increase of temperature leads to a failure state by the total breakdown of the equipment and causes a cooling problem, causing expensive and large losses.

Our work proposes as a solution to this problem, the design of a mill supervision interface and the implementation of a mill failure prediction and classification system. The missions of this system are subdivided into two main activities: the prediction of the future evolution of the degradation of the temperature and the classification of the predicted state, according to the various known functional states (normal, degraded and breakdown), in view of diagnostic assistance via the interface.

\section{PROGNOSIS OF CARBOMILL FAILURES BY NEURO-FUZZY APPROACH}

Current technological advances are having a growing influence in the industrial environment, reflected in the complexity of new industrial systems, in search of satisfactory overall performance [3]. Thus, the processes dedicated to the control and the optimization of the systems during their operation benefited from the contribution of new tools and new methods of control-command, supervision and maintenance aiming to anticipate on the future behavior of a system. For this purpose, the prognosis generally aims to either determine the lifespan of a RUL (Remaining Useful Life) system [11], [12], [13] and [14] which is the probability that the system will work for a certain time [3].

The prognosis of industrial systems can be based on three approaches: the model-based approach, the experiencebased approach and the data guided approach [4], [15] and [16]. The increased complexity of industrial equipment like the malt mill makes it difficult to obtain a physical model. Oherwise, the availability of sensor measurements will allow us to work with the data guided approach for the rest of our work. From this point of view, the prognosis is generally realized by artificial intelligence methods such as neural networks and fuzzy logic [17] and [18].

While neural networks are interesting for recognizing models, they unfortunately can't explain how they reach their decisions (black box). It is the same for fuzzy systems that can reason with inaccurate information, they are very interesting to 
explain their decisions, but can't automatically acquire the rules they use to make these decisions. The use of both neural networks and fuzzy logic to reap the benefits of the two methods has resulted in the neuro-fuzzy approach [19] and [20]. The neuro-fuzzy systems present the strengths of the two tools of artificial intelligence and above all allow to overcome their limits.

Various associations of fuzzy logic approaches and artificial neural networks have been developed in recent years, bringing innovations in several areas, particularly time series prediction. Beyond the concepts of cooperative neuro-fuzzy networks [21] competing neuro-fuzzy networks [22], the hybrid neuro-fuzzy network [23] and [24] draws better because, taking advantage of the combined use of fuzzy systems associated in series and in parallel. Several classifications can thus be distinguished: NEFCLASS, NEFDIAG, NEFCON, NEFPROX and ANFIS. The first-class Takagi-Sugeno ANFIS neuro-fuzzy network (outputs of subsystems are linear combinations of inputs) [25], [26] [27] and [28] is an effective tool for approximating non-linear functions. It has the advantage of having an adaptive structure to all the input data of the network. Thus, during learning, the set of observation values automatically modifies the structure of the network for a better approximation.

\section{CLASSIFICATION OF PREDICTED TEMPERATURES IN OPERATING MODES}

The knowledge of the operating mode of a system from the evolution of the significant parameters, makes it possible to alert the operator about the functional state of the system. This allows him to initiate maintenance actions, in order to reduce unavailability. Indeed, Carbomill temperatures are grouped into classes corresponding to each state of operation (normal, degraded and breakdown). The "degraded" state is characterized by a threshold corresponding to a temperature of $60{ }^{\circ} \mathrm{C}$ above which the system is declared faulty.

The implementation of a classification system in our case, allows to assign to each temperature predicted by the neurofuzzy network, a state of operation. There are three main methods of classification: methods based on knowledge. They are used when a sufficient knowledge is available for a process dynamic. This method exploits the knowledge that the experts hold of the process, to assign an observation to a state of operation; internal methods exploit the physical or analytical model of the process. They make it possible to analyze a certain number of numerical values resulting from the analytical model and compare them with data measured on it. Finally, there are the external methods. They are adapted when the only information available is the signals from the sensors positioned on the equipment.

\section{CONTROLLING COMMUNICATION BETWEEN MONITORING, PREDICTION AND CLASSIFICATION SYSTEMS USING ETHERNET TCP / IP PROTOCOL}

A computer protocol is a machine-machine language by which devices can communicate in a unified language, so as to understand each other and to exchange informations through various channels. For this purpose, to ensure the communication between the PLC and the HMI, where will be represented the graphical interface, we chose the TCP / IP Ethernet protocol. It will also ensure communication between the PLC and the prediction and classification systems.

The Modbus TCP / IP industrial LAN is a variant of the Modbus protocol that transfers data between several devices via the TCP / IP protocol, according to a client / server architecture. It represents the key point for transferring information from the prediction and classification systems under Matlab R2013a to the Unity Pro Premium PLC. Each device been identified by an IP address, the client (Matlab) through a request frame, will require information from the server. Then the server (Unity Pro) will execute the request frame and returns a response frame to give it the requested information (if necessary). Most of the communication between the different applications of our project is summarized in Figure 3. 


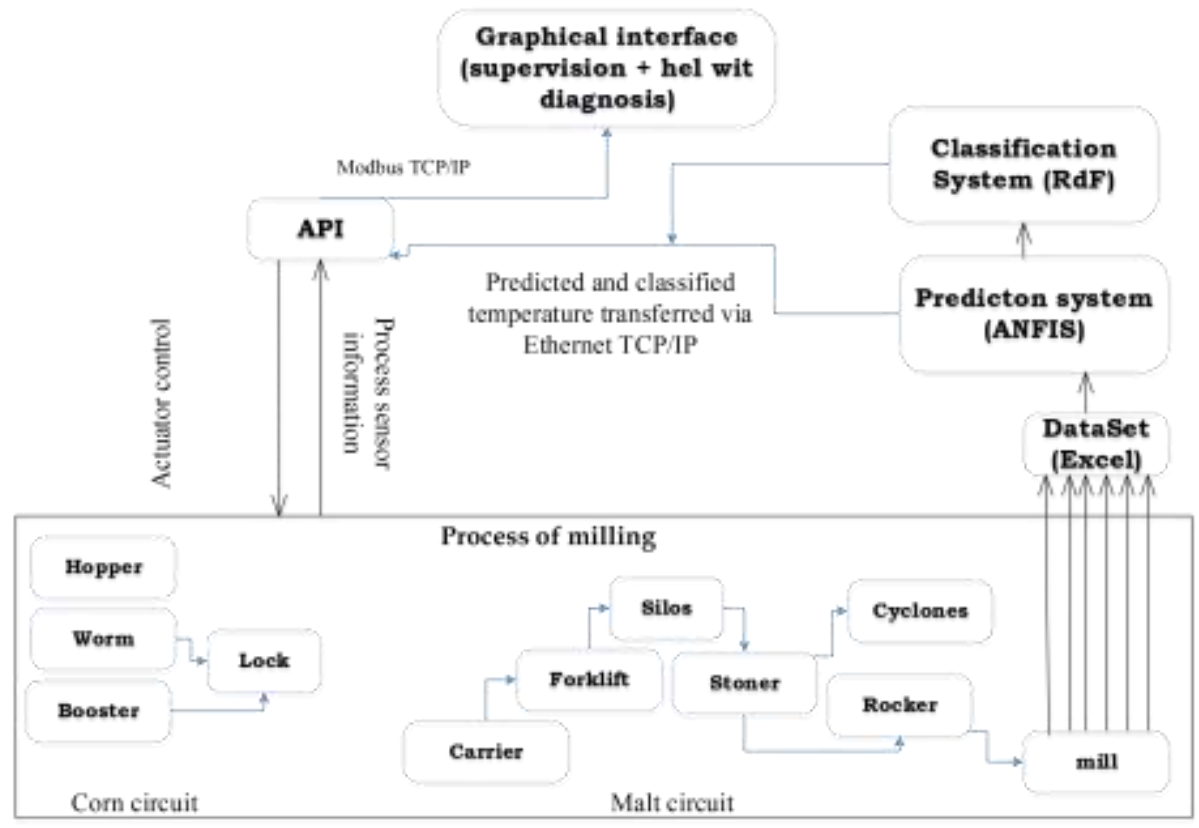

Figure 3: Synoptic of the AI-PLC combinatorial approach

\section{RESULTS AND DISCUSSIONS}

The design of the mill supervision interface was realized with Vijeo Designer v4.6.0 software, which allows to realize the graphic design of the milling process. Then the interface will be loaded into a Magelis XBTGK 2330 operator dialog. The control of the graphic interface is ensured by a Premium PLC, programmed on Unity Pro XL V6.0. Then the PLC will take into account all the operating parameters of the mill. Indeed, the graphical supervision interface consists of 10 main views and three secondary views.

\subsection{Graphical interface for supervision and diagnostic assistance}

The "Silage" and "Unsilage" views (figure 4 and 5) represent the synoptics silage and unsilage operations of the malt circuit. The malt is stored in the silo during silage. Counter to unsilage, which consists to remove from the silo the malt used for milling.

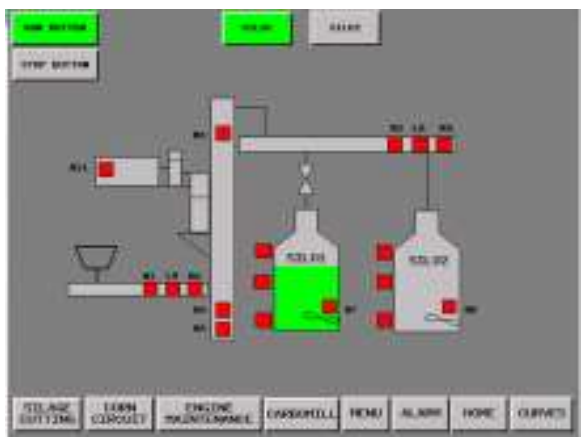

Figure 4 : Presentation view "Silage"

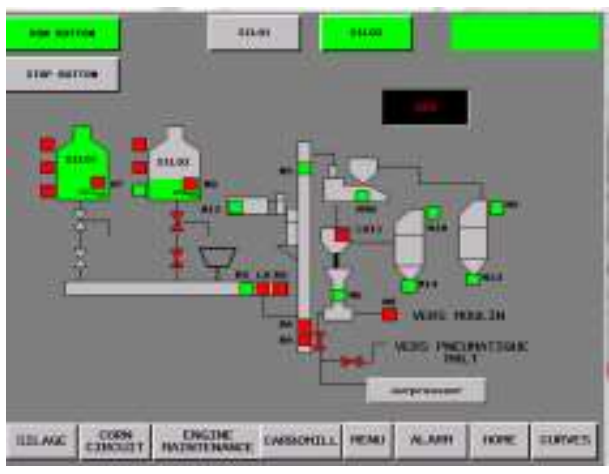

Figure 5 : Presentation view «Unsilage »

The "Corn Circuit" view (figure 6) represents the synoptic of this circuit operations. This is a fairly simple circuit that is justified by the few numbers of components on the graphical interface. The "Alarm" view (figure 7) shows the defects of the equipment of the mill, which are represented in red. Once a fault has been acknowledged, its color changes to blue. 


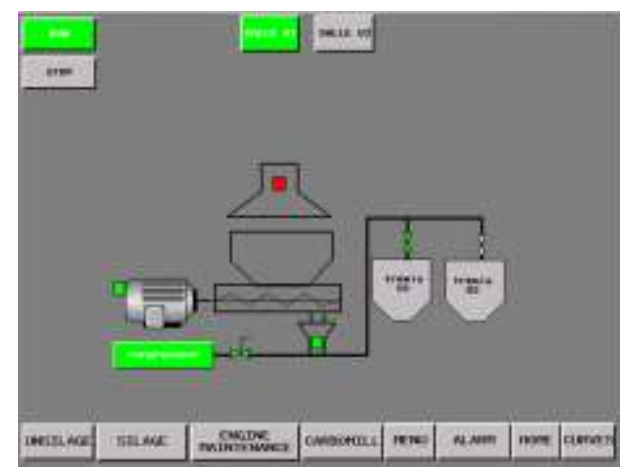

Figure 6: Presentation view « Corn Circuit »

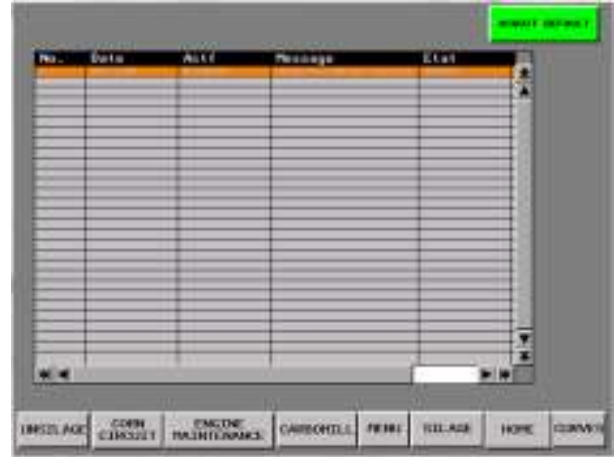

Figure 7 : Presentation view « Alarm»

The ' cmymle iviantenance (ngure of anu 1 mile stales of engines" views (figure 9) are used to maintain all engines in the mill. Indeed, the operator must be able to enter the hour of operation before maintenance of each engine. The operator must be warned by an alarm, 170 hours before the date of actual maintenance. This allows to prepare the formalities for preventive actions. The time between failures counter thus records the total hours of operation of the engines. The breakdown time counter, on the other hand, indicates the total hours of shutdown for each engine.

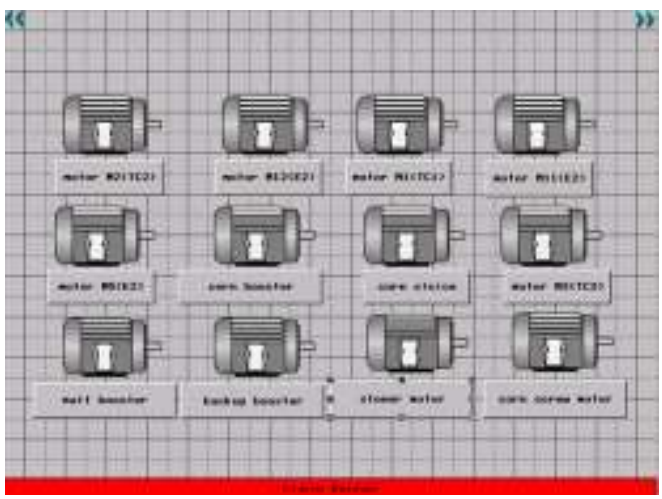

Figure 8 : Presentation view « Engine Maintenance»

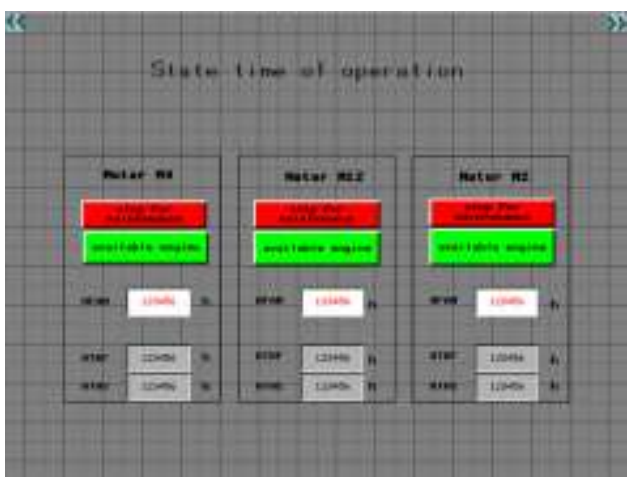

Figure 9: Presentation view « Time states of engines »

The "Carbomill" view (figure 10) shows the operation of the grinder and the windows such as: "beer recipe", "set point indicators" and many more. The "Diagnosis help" view (figure 11) shows the probable causes of degradation of the mill temperature for an abnormal operating mode. The diagnosis help is taken from a Failure Mode and Effect Analysis (FMEA) study not presented in this article.

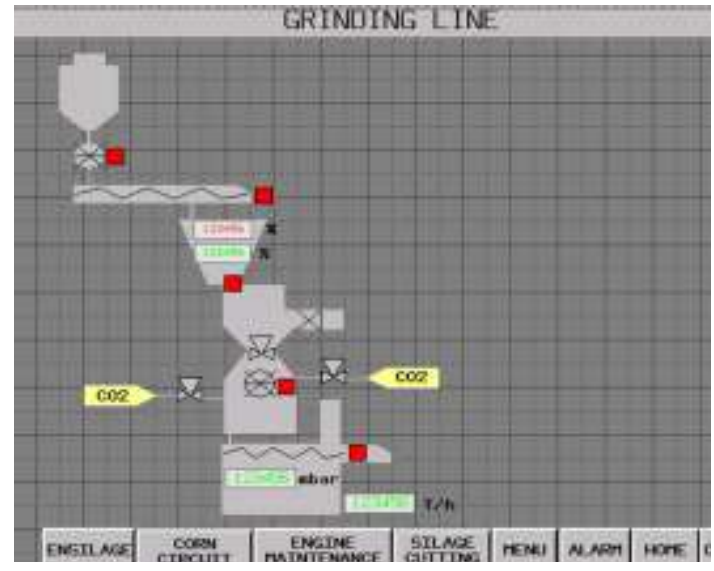

Figure 10 : Presentation view «Carbomill »

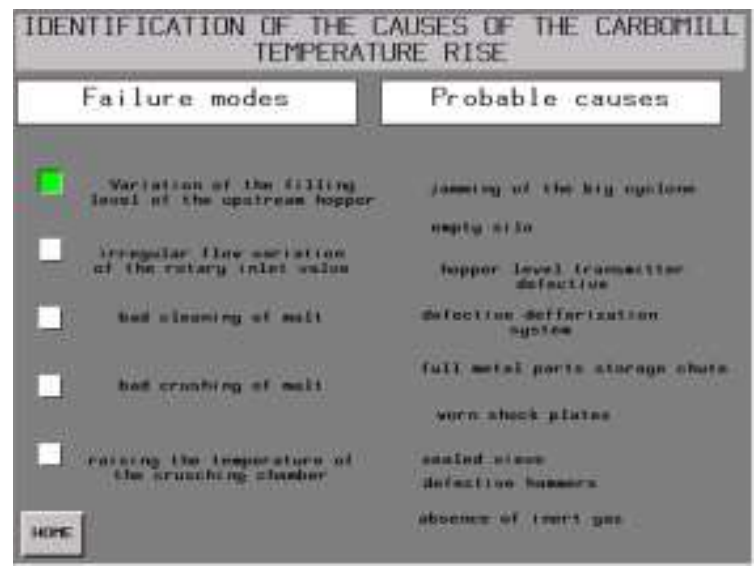

Figure 11: Presentation view « Diagnosis help » 


\subsection{Evolution of Carbomill temperature and estimation of RUL}

The Carbomill temperature signal is not stationary (Figure 12), which justifies the complexity of the mill. For this purpose, the prediction system is responsible for predicting this change of temperature over time. This temperature change will then be plotted in the interface by a trend line. The trend curve is used to represent the variation of the values of a variable over time. The trend curve represents 03 channels (or variables) namely: the temperature predicted by ANFIS, the upper temperature of the degraded state which is $60{ }^{\circ} \mathrm{C}$ and the lower temperature of the breakdown state being $66{ }^{\circ} \mathrm{C}$. The highlighting of an abnormal temperature, leading to the "breakdown" state by the predictive system, triggers the calculation of the Remaining Useful Life (RUL) given by the following equation (1):

$$
R U L=t_{2}-t_{1}
$$

Where:

$\boldsymbol{t}_{2}$ : instant when the actual temperature crosses the upper limit of the degraded temperature;

$\boldsymbol{t}_{\mathbf{1}}$ : instant start of prediction of a temperature corresponding to the breakdown state;

$\boldsymbol{R} \boldsymbol{U} \boldsymbol{L}$ : Lifetime before occurrence of failure.

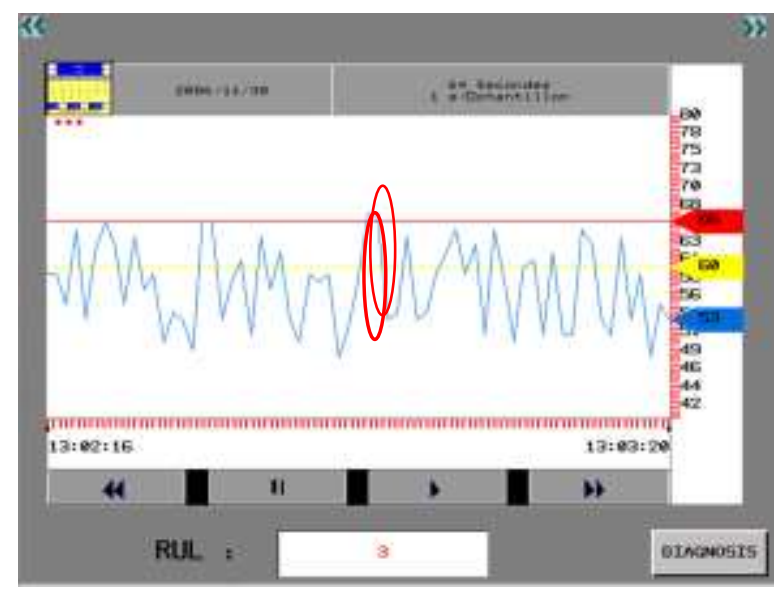

Figure 12: Plot of the evolution of the temperature and calculation of the RUL

\subsection{Construction of the neural network classification and performance evaluation}

The input layer has six inputs: threshold level, valve opening percentage, mill amperage, crushing rate, $\mathrm{CO}_{2}$ pressure and predicted temperature. The first hidden layer has 36 neurons and the second has 3 neurons, with an output layer of three neurons representing the three classes (states) of operation (Figure 13).

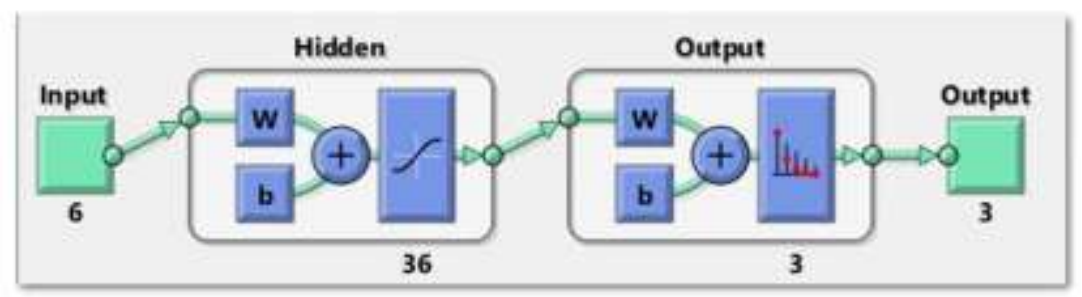

Figure 13: Construction of the Feedforward neural network for learning

The two hidden layers of neural network allows us to obtain the best classification results. Indeed, the number of neurons of the first hidden layer makes the network flexible and thus bring near the relationship between the variables of the inputs and 
target outputs. The assignment of each observation couple to a class is estimated by the membership probability provided by the Softmax function.

The evaluation of the network performance is carried out by drawing the confusion matrix (Figure 13). This matrix presents the rate of good and bad classifications. The plot of the Receiver Operating Characteristic (ROC) curve also reveals the classification probabilities of false positives and true positives for each class of operation.

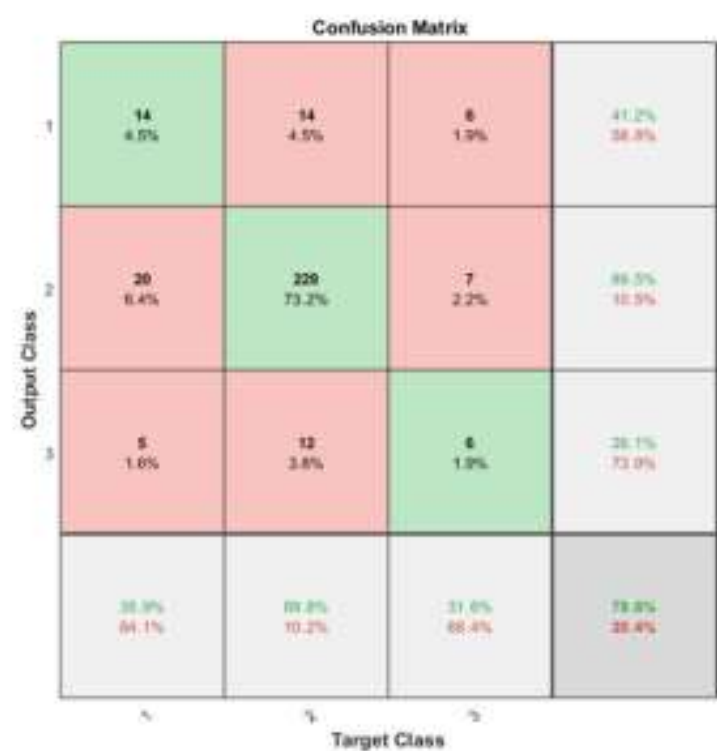

Figure 13: Confusion matrix of desired and actual output classes

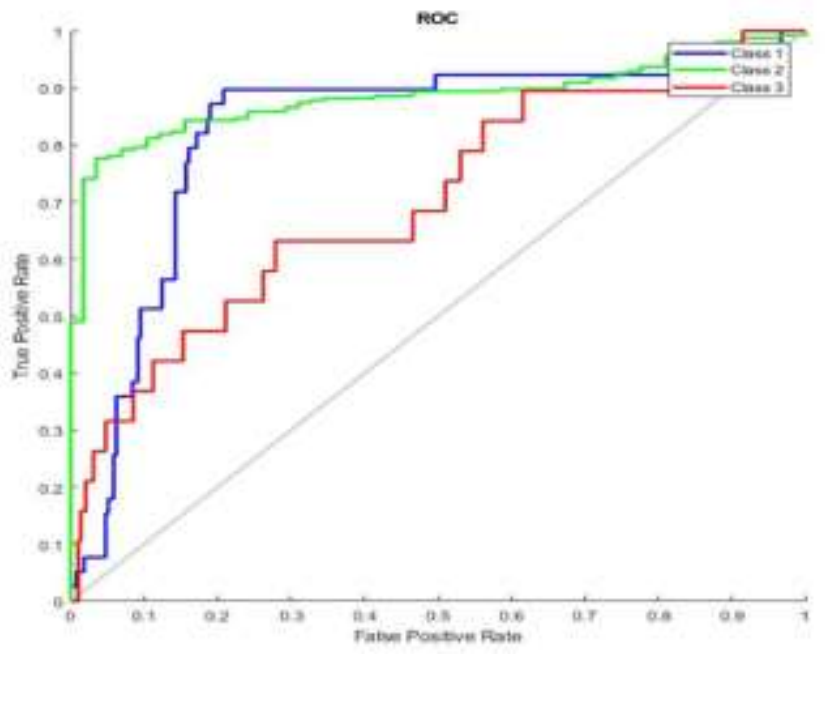

Figure 14: The ROC curve of the output classes of the classification network

The confusion matrix (figure 13) presented makes it possible to evaluate the performance of the classification network and to evaluate the adjustment of the weights, by the cross-entropy cost function. The network successfully classifies just 14 observations for class 1; 229 observations for class 2 and 6 observations for class 3 . However, it is mistaken about the classification of 20, then of 5 observations as belonging to class 2 and 3, even though they actually belong to class 1 . In the same way, with the classification of 14 observations, then of 12 observations as belonging to class 1 and 3 , while they actually belong to class 2 . Similarly, with the classification of 6 observations, then of 7 observations as belonging to class 1 and 2 while they actually belong to class 3 . The overall performance rate of the network is thus estimated at $79.6 \%$.

The ROC curve (figure 14) presents the network classification probabilities for "True Positive Rate" and "False Positive Rate". Indeed, the "True Positive Rate" represent the set of observations correctly classified to their mode of operation. On the other hand, the "False Positive Rate" represents the observations assigned to a class even though they are not part of it. In figure 14, we find that the area occupied by Class 3 (breakdown) approaches the right, so the network had difficulties to classify the observations of this class. The same is true for class 1 (degraded) observations.

\section{CONCLUSION}

In this article, we presented a graphical interface created under Vijeo Designer and controlled by a PLC programmed on Unity Pro XL. The graphical interface actually fulfills all expected control-command and diagnosis assistance specifications. For the prediction system, the ANFIS neuro-fuzzy network was used as a prediction tool. It provides good results at the end of its learning evaluated by an RMSE cost function of 0.2142. However, the performance of the generalization of this network on test data could have given good results if the data set was more consequent. The predicted temperature classification system, on the other hand, provides good overall results in assigning a predicted temperature value to a state of operation. We thus observe a classification percentage of about 79.6\%. However, we find that the basis of our Carbomill temperature forecast, which is based only on the ANFIS neuro-fuzzy network, is not evolving in the face of new observations. Our work continues with the aim of developing a prediction system using evolutionary algorithms, making it robust and scalable in the face of new data to enable online learning. 


\section{REFERENCES}

[1] Villani, C. Giving meaning to Artificial Intelligence for a European national strategy, Parliamentary Mission Report made from September 08, 2017 to March 08, 2018, France.

[2] Cisco, Transform your business with artificial intelligence. Cisco, 2018.

[3] El Koujok, M. Contribution to industrial prognosis: integration of trust in a predictive neuro-fuzzy model. Unpublished PhD thesis, the University of Franche-Comté, 2010, France.

[4] Muller, A. Contribution to the predictive maintenance of production systems by formalizing a prognostic process. Unpublished PhD thesis, University Henri Poincaré of Nancy I, 2005, France.

[5] Traoré, M.A. Adaptive Supervision and Failure Prognosis for Predictive Maintenance of Complex Evolutionary Systems. Unpublished PhD thesis, University of Lille 1, 2010, France.

[6] Nzie, W., Samon, C.J.B. and Balinguin, B. (2014) 'Modeling of a prognostic tool for the optimization of a predictive preventive maintenance policy’. European Scientific Journal, july, 2014 edition, vol. 10, № 21, pp 226-238.

[7] Nzeneu Mbakakeu, S.G. Conception of a beer manufacturing System of the breweries of Yaounde (Cameroon). Unpublished thesis, Faculty of Industrial Engineering, University of Douala, 2018, Cameroon.

[8] Sandel, O. Intelligent interface model for communication terminals. Unpublished PhD thesis, the University of Strasbourg 1Louis Pasteur, 2002, France.

[9] Adam, E. Multi-Agent Organization Model for Helping Cooperative Work in Business Processes: Case study of Complex Administrative Systems. Unpublished PhD thesis, University of Valenciennes and the Hainaut-Cambresis, 2000, France.

[10] Zhang, N. Detection and isolation of faults based on differential flatness application to atmospheric gear. Unpublished PhD thesis, University of Toulouse 1,2010, France.

[11] Nguyen, T.B.L. (2016) Statistical Approach to the Prognosis of Failure : Application to the Semiconductor Industry. Unpublished PhD thesis, the University of Aix-Marseille, France.

[12] Sanchez, R.E.S. (2016) Contribution to lifetime of PEMFC fuel cell systems. Unpublished PhD thesis, the University of Quebec at Trois Rivières, Canada.

[13] Aggab, T., Vrignat, P., Avila, M. and Kratz, F. 'Establishment of a degradation prognosis in a subservient system'. Paper Presented at the 20th Congress on Risk Management and Dependability. 11-13 october 2016. Saint-Malo, France.

[14] Aggab, T., Kratz, F., Vrignat, P. and Avila, M. 'Remaining Useful Life prediction method using an observer and statistical inference estimation methods'. Annual conference of the prognostics and health management society 2017. October 2017, St. Petersburg, Florida, United States.

[15] Debbah, Y Development of a prognostic tool for the maintenance of mechanical systems. Unpublished PhD thesis, the University of the Mentouri-Constantine Brothers, 2018, Algeria.

[16] Nguyen, D.N. Contribution to probabilistic approaches for the prognosis and maintenance of controlled systems. Unpublished PhD thesis, the University of Technology of Troyes, 2015, France.

[17] Chevrie, F. and Guély, F. The Fuzzy logic. Technical paper No. 191, Schneider Group, march 1998 edition.

[18] Bazoge, C. and Dias Lopez, A. ORSA : Contribution of Fuzzy Logic in Non-Life Management actions modeling. Unpublished thesis, Center of Actuarial Studies, 2017, Paris, France.

[19] Benaicha, S., Mouss, H., Bencherif, F. and Srairi, F. (2013) Neuro-fuzzy control of a clinkerisation system. Paper Presented at the International Conference on Electronics \& Oil : From theory to applications. 05-06 march 2013. Ouargla, Algeria.

[20] Taleb, A. Contribution of neuro-fuzzy networks and genetic algorithms to speech recognition. Unpublished PhD thesis, University of Science and Technology of Oran Mohamed Boudiaf, 2010, Algeria.

[21] Palluat, N. (2006) Dynamic monitoring methodology using temporal neuro-fuzzy networks. Unpublished PhD thesis, the University of Franche-Comté, France. 
[22] Benaissa, M.O. Contribution to the study of energy transfer in photovoltaic systems. Unpublished PhD thesis, University of Djillali Liabes of Sidi-Bel-Abbes, 2018, Algeria.

[23] El-Hadi, R. (2003) Automatic generation and optimization of fuzzy inference systems. Unpublished PhD thesis, University of Paris XII Val de Marne, France.

[24] Zemalache Meguenni, K. (2006) Control of an under-powered system : Application to a four-propeller drone. Unpublished $\mathrm{PhD}$ thesis, University of Evry Val d'Essome, France.

[25] Gouriveau, R., El Koujok, M. and Zerhouni, N. (2007) Specification of a neuro-fuzzy system for predicting medium-term failures. Paper Presented at the French Meetings on fuzzy Logic and Applications, LFA 2007. November 2007, Nîmes, France.

[26] Adeline, R., Gouriveau, R. and Zerhouni, N. (2008) 'Prognosis of failures: Control of the prediction error'. Paper Presented at the 7th International Conference on MOdelling and Simulation, MOSIM'08. March 2008, Paris, France.

[27] Dragomir, O., Gouriveau, R. and Zerhouni, N. (2008) Industrial prognosis: study of the prediction error of the ANFIS system. Paper presented at the International Francophone Conference of Automation, CIFA'08. September 2008, Bucharest, Romania.

[28] Kebe, B., Ba, O., Niang, B. and Thiaw, L. (2017) 'Study, synthesis and implementation of a neuro-fuzzy controller for turbo generator command'. Review of the CAMES-Applied Sciences and the Engineer. Vol. 2(2), pp 46-53. 